\title{
Rescue Mask with Prefabricated Leak for Reduction of Accidental Stomach Inflation During Lay Rescuer Ventilation
}

\author{
Nicole Schnider ${ }^{1}$, Klaus W. Graetz ${ }^{1}$ and Till S. Mutzbauer*,2 \\ ${ }^{I}$ Department of Craniomaxillofacial and Oral Surgery, University Hospital and Dental Center Zuerich, Frauenklinik- \\ strasse 24, CH-8091 Zuerich, Switzerland \\ ${ }^{2}$ Mutzbauer\&Partner, Maxillofacial Surgery and Anesthesiology, Zuerich; Tiefenhoefe 11, CH-8001 Zuerich, Switzerland
}

\begin{abstract}
Objective: To evaluate the efficacy of a simple method to minimize gastric inflation in unprotected airways during resuscitation procedures.

Methods: Ventilation of an airway simulator by use of a hose extended mask with leak and without leak and with or without additional oxygen (15 litres per minute). Experimental settings with artificial lung at a compliance $=300 \mathrm{ml} / \mathrm{kPa}$ and resistance $=0,5 \mathrm{kPa} / 1 / \mathrm{s}$. Lower oesophageal sphincter pressure $=0,5 \mathrm{kPa}$.

Results: Tidal volumes were significantly lower with integrated leak than with the leak proof mask (medians: 406 vs. $681 \mathrm{ml}$ ), gastric inflation was significantly reduced by use of the mask with leak (medians: $2300 \mathrm{ml}$ vs. $7300 \mathrm{ml}$ ) and inspiratory oxygen fraction was significantly higher when oxygen was added.

Conclusion: Gastric inflation may markedly be reduced, however not excluded, when a mask with a prefabricated leak is used instead of achieving a leak proof mask ventilation technique. With additive oxygen only the inspiratory oxygen fraction will be significantly higher which supports the benefit of additional oxygen.
\end{abstract}

Keywords: Lay Rescuer Ventilation, Rescue Ventilation Mask, Stomach Inflation, Leak.

\section{INTRODUCTION}

Bag-valve-mask ventilation with an unprotected airway during cardiopulmonary resuscitation (CPR) is often performed too aggressively, when inexperienced lay rescuers are observed during mannequin training. Thereby, excessive flow velocities and peak airway pressures may cause stomach inflation [1].

Regurgitation and subsequent aspiration and a decrease of respiratory compliance can result [2]. Considering these undesired effects it is necessary to minimize likelihood of gastric inflation.

Earlier studies show that for example regurgitation during CPR can be reduced by use of a laryngeal mask airway, a laryngeal tube or a new supraglottic airway device (I-gel) instead of a bag-valve-mask device [3-5]. Placement of airway adjuncts such as a laryngeal tube requires training and regular practice to achieve competence. Consequently, lay rescuers have to deal with an unprotected airway [6].

The aim of this study was to evaluate the efficacy of a simple method to minimize gastric inflation in unprotected airways during resuscitation procedures. The first hypothesis to test was that lower gastric inflation volumes will result when a mask with a prefabricated leak is used in comparison to a leak proof mask. The second hypothesis was that addi-

*Address correspondence to this author at the Mutzbauer\&Partner, Maxillofacial Surgery and Anesthesiology, Zuerich; Tiefenhoefe 11, CH-8001 Zuerich, Switzerland; Tel: +41 44211 1465; Fax +41 44212 4085;

E-mail:mutzbauer@gmail.com tional oxygen connected to the mask with prefabricated leak does not increase gastric inflation volumes.

\section{MATERIALS AND METHODOLOGY}

The experimental protocol used in this study was reviewed and approved by the local ethics committee. 17 dental students in their last year of education volunteered to participate. The subjects did not have been trained in CPR for at least six months before, but all had been well educated in CPR during their first two clinical years in dental school.

All participants were asked to ventilate the airway simulator. This simulator consisted of an upper airway, an artificial lung (SmartLung ${ }^{\mathrm{TM}}$, imtmedical ag, Buchs SG, Switzerland) and an oesophagus/stomach system (corrugated tube connected to the upper airway by a $\mathrm{T}$ piece, submersed under a $5 \mathrm{~cm}$ water seal in a plastic infusion bottle). The lung was connected to a ventilation training head (VBM medical, Sulz aN, Germany) (Fig. 1).

Ventilation was achieved by use of a face mask (Ambu mask size 3, Ambu, Glostrup, Denmark). Oxygen could be inflated directly into the mask by a flexible silicone tube. The oxygen tube was attached to the gas sampling port of the breathing filter (Medisize Barr-Vent S, Medisize bv, Hillegom, The Netherlands) connected to the mask. The filter was connected to a mouth piece by an interposed $\mathrm{T}$ piece. An ISO $15 \mathrm{~mm}$ endotracheal tube connector size 8.0 (Dräger, Lübeck, Germany) was attached to the remaining port of the $\mathrm{T}$ piece to achieve a standardized leak. As a control device, the mouthpiece was directly connected to the breathing filter - without the T piece. 


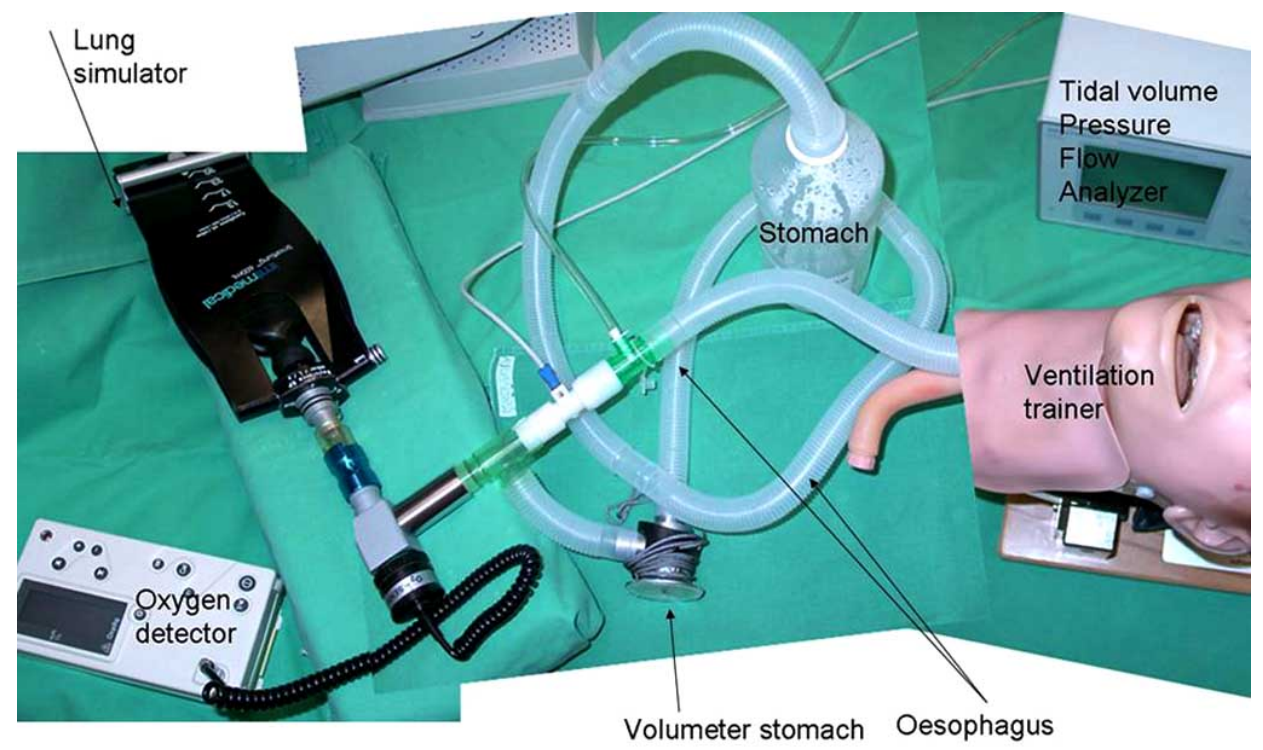

Fig. (1). Experimental setting: artificial lung adjusted to compliance $\mathrm{C}=30 \mathrm{ml} / \mathrm{mbar} ; 300 \mathrm{ml} / \mathrm{kPa}$ and resistance $\mathrm{R}=5 \mathrm{mbar} / \mathrm{l} / \mathrm{s} ; 0.5 \mathrm{kPa} / \mathrm{l} / \mathrm{s}$. Water seal W with a water column of $5 \mathrm{~cm}$ with a submerged artificial oesophagus simulating a lower oesophageal sphincter pressure of 5 mbar; $0.5 \mathrm{kPa}$. Tidal volume/pressure/flow analyzer for data acquisition (and storage to personal computer) connected to pressure line and flow detector. Oxygen detector positioned at lung entrance.

The study device is illustrated in Fig. (2).

Peak pressure (PMAX), inspiratory tidal volume (TVIN), maximum inspiratory flow (MAXF) and gastric inflation volumes (stomach volume) over 10 ventilation cycles as well as the inspiratory oxygen fraction were recorded (Acutronic Medical Systems AG, Hirzel ZH, Switzerland).

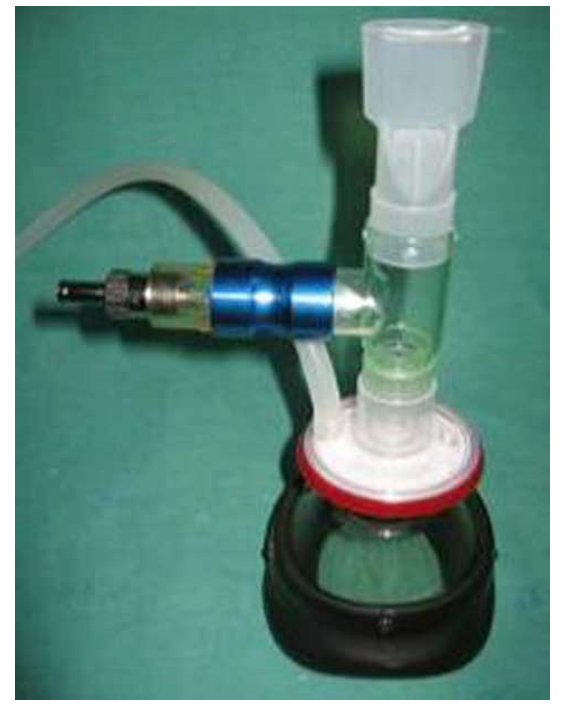

Fig. (2). Experimental ventilation device consisting of an AMBU mask size 3, a breathing filter connected to a mouth piece via a $\mathrm{T}$ piece. The system included a leak generated by an open Dräger ISO 15 connector size 8.0. Oxygen could be inflated via the gas detector port of the breathing filter.

\section{Test Procedure}

The volunteers were only instructed to treat the experimental model as an adult in respiratory arrest and to ventilate the device via the mouth piece and the face mask ten times in a way they thought to be adequate. Two main settings were given: Ventilation with and without additional oxygen (15 liters per minute). Both settings were used either combined with the leak or without leak. The order of the sequences was randomized.

The participants were able to see the movements of the artificial lung and they could hear the bubbles under the water seal simulating the lower oesophageal sphincter/stomach system during ventilation.

\section{Statistics}

Median values of the distribution of peak airway pressure, inspiratory tidal volume, maximum inspiratory flow and stomach inflation volume were calculated by use of the respective device achieved by each volunteer during each breath.

All data obtained from each participant by use of the different systems were compared by exact Wilcoxon test statistics for matched pairs and Friedman test statistics, respectively, using the Excel add-in Analyse-it (Analyse-It Software Ltd., Leeds, England, UK).

\section{RESULTS}

An overview of the results is given in Table $\mathbf{1}$.

With integrated leak (L), tidal volumes were significantly lower than with the leak proof mask (T) (medians: $406 \mathrm{ml}$ vs. $681 \mathrm{ml}$ ). Moreover, gastric inflation was significantly reduced (medians: $2300 \mathrm{ml}$ vs. $7300 \mathrm{ml}$ ) by use of the mask with leak. The range of peak inspiratory airway pressures and maximum inspiratory flow values was higher by use of the leak proof mask compared to the mask with leak.

Tidal volumes, peak inspiratory airway pressures, maximum inspiratory flows and gastric inflation volumes measured by use of the mask-leak combination with additional oxygen $(\mathrm{O} 2)$ did not differ from the respective parameters without extra oxygen $(\mathrm{Br})$. 
Table 1. Respiratory Parameters and Stomach Inflation Volume Achieved by a Rescue Mask with a Prefabricated Leak

\begin{tabular}{|c|c|c|c|c|}
\hline \multicolumn{5}{|c|}{ TVIN (inspiratory tidal volume) [ml] } \\
\hline $\max$ & 2902 & 934 & 979 & 900 \\
\hline $75 \% \mathrm{Q}$ & 1362 & 623 & 690 & 774 \\
\hline median & 681 & 406 & 452 & 592 \\
\hline $\mathrm{p}[\mathrm{L}$ vs Br vs O2] & & \multicolumn{3}{|c|}{0.29} \\
\hline $\mathrm{p}[\mathrm{T}$ vs $\mathrm{L}]$ & \multicolumn{2}{|c|}{$<0.0001$} & & \\
\hline \multicolumn{5}{|c|}{ Stomach volume [ml] } \\
\hline $\max$ & 20900 & 8200 & 9200 & 9500 \\
\hline $25 \% \mathrm{Q}$ & 4000 & 1100 & 2400 & 4000 \\
\hline $\min$ & 2200 & 0 & 0 & 0 \\
\hline $\mathrm{p}[\mathrm{L}$ vs Br vs O2] & & \multicolumn{3}{|c|}{0.05} \\
\hline $\mathrm{p}[\mathrm{T}$ vs L $]$ & \multicolumn{2}{|c|}{$<0.0001$} & & \\
\hline \multicolumn{5}{|c|}{ PMAX (peak pressure) [mbar] } \\
\hline $\max$ & 31 & 18 & 15 & 17 \\
\hline $75 \% \mathrm{Q}$ & 15 & 9 & 10 & 11 \\
\hline median & 7 & 5 & 7 & 8 \\
\hline $75 \% \mathrm{Q}$ & 82.13 & 60.89 & 69.19 & 63.06 \\
\hline Median & 50.04 & 47.99 & 51.71 & 50.2 \\
\hline $25 \% \mathrm{Q}$ & 40.235 & 29.005 & 41.515 & 39.81 \\
\hline Min & 26.63 & 15.33 & 8.35 & 9.54 \\
\hline $\mathrm{p}[\mathrm{L}$ vs Br vs O2] & & \multicolumn{3}{|c|}{0.55} \\
\hline $\mathrm{p}[\mathrm{T}$ vs L $]$ & \multicolumn{2}{|c|}{0.0003} & & \\
\hline \multicolumn{5}{|l|}{$\% \mathrm{O} 2$} \\
\hline $\max$ & & & 21 & 80 \\
\hline $75 \% \mathrm{Q}$ & & & 20 & 48 \\
\hline median & & & 19 & 43 \\
\hline $25 \% \mathrm{Q}$ & & & 18 & 40 \\
\hline $\min$ & & & 14 & 32 \\
\hline $\mathrm{p}[\mathrm{Br}$ vs O2] & & & \multicolumn{2}{|c|}{$<0.0001$} \\
\hline
\end{tabular}

Inspiratory tidal volume (TVIN), peak pressure (PMAX) and maximum inspiratory flow (MAXF) observed per breath - as well as gastric inflation volume (stomach volume) observed during ten ventilations of an artificial lung at a compliance $\mathrm{C}=30 \mathrm{ml} / \mathrm{mbar} ; 300 \mathrm{ml} / \mathrm{kPa}$ and a resistance $\mathrm{R}=5 \mathrm{mbar} / \mathrm{l} / \mathrm{s}(0.5 \mathrm{kPa} / 1 / \mathrm{s})$ at a simulated lower oesophageal sphincter pressure of $5 \mathrm{mbar}(0.5 \mathrm{kPa}) ; 17$ volunteers. 
Only the inspiratory oxygen fraction was significantly higher when oxygen was added.

\section{DISCUSSION}

The distribution of inflated gas volumes between lungs and stomach in the unprotected airway depends on patient variables such as lower oesophageal sphincter pressure, airway resistance and respiratory system compliance as well as the victim's head position and the ventilation technique chosen by the rescuer [7].

Since patient variables can not be influenced by rescuers it is necessary to focus on technique and equipment to achieve better results.

Other studies emphasize the importance of developing new technical devices that reduce stomach inflation by limiting tidal volume $[8,9]$.

The main result of the present study is that gastric inflation may markedly be reduced when a mask with a prefabricated leak is used instead of achieving a leak proof mask ventilation technique. A mask leak will reduce peak pressure and flow during ventilation. Additional oxygen inflated into a leaking mask, will hardly increase pressure and flow and, thereby, gastric inflation and tidal volume. Only the inspiratory oxygen fraction will be significantly higher. That supports the benefit of additional oxygen administered at a high flow [6].

\section{METHODOLOGICAL LIMITATIONS}

This model cannot exactly reproduce a patient in the situation of respiratory arrest.

There was no stomach compliance, as the stomach was represented by the air downstream to the water seal. It cannot be excluded that the experimental setting has favoured or reduced gastric inflation or pulmonary ventilation. Bubbling of the water seal could be heard by the participants and might have influenced the ventilation technique as well.

\section{CONCLUSION}

A mask with a prefabricated leak used in a simulator shows a significant reduction of gastric inflation, even if gastric inflation can not be excluded.
Tidal volume will be significantly lower with a leaking mask. This would be outweighed by a reduced gastric inflation. As lay rescuers cannot be trained to create a leak during mask ventilation, manufacturing masks with a prefabricated leak may contribute to reduce the risk of accidental gastric inflation during cardiopulmonary resuscitation.

\section{REFERENCES}

[1] Von Goedecke A, Wagner-Berger HG, Stadlbauer KH, et al. Effects of decreasing peak flow rate on stomach inflation during bagvalve- mask ventilation. Resuscitation 2004; 63(2): 131-6.

[2] American Heart Association. Guidelines for cardiopulmonary resuscitation and emergency cardiovascular care: Part 4: Adult basic life support. Circulation 2005; 112(suppl 24): IV-19-34.

[3] Stone BJ, Chantler PJ, Baskett PJF. The incidence of regurgitation during cardiopulmonary resuscitation: a comparison between the bag valve mask and laryngeal mask airway. Resuscitation 1998; 38(1): 3-6.

[4] Stohler FC, Becker MF, Tabacek G, Drommer RB, Mutzbauer TS. Alternative concept of ventilation during cardiopulmonary resuscitation(CPR) in dental chairs. Schweiz Monatsschr Zahnmed 2007; 117(8): 814-9.

[5] Beylaco L, Bordes M, Semjen F, Cros AM. The I-gel, a single-use supraglottic airway device with a non-inflatable cuff and an esophageal vent: an observational study in children. Acta Anaesthesiol Scand 2009; 53(3): 376-9.

[6] Von Goedecke A, Keller C, Voelckel WG, et al. Mask ventilation as an exit strategy of endotracheal intubation. Anaesthesist 2006; 55(1): 70-9.

[7] Wenzel V, Idris AH, Dörges V, et al. The respiratory system during resuscitation: a review of the history, risk of infection during assisted ventilation, respiratory mechanics, and ventilation strategies for patients with an unprotected airway. Resuscitation 2001; 49(2): 123-34.

[8] Paal P, Falk M, Sumann G, et al. Comparison of mouth-to-mouth, mouth-to-mask and mouth-to-face-shield ventilation by lay persons. Resuscitation 2006; 70(1): 117-23.

[9] Wagner-Berger HG, Wenzel V, Stallinger A, et al. Decreasing peak flow rate with a new bag-valve-mask device: effects on respiratory mechanics, and gas distribution in a bench model of an unprotected airway. Resuscitation 2003; 57(2): 193-9.

(C) Till S. Mutzbauer; Licensee Bentham Open.

This is an open access article licensed under the terms of the Creative Commons Attribution Non-Commercial License (http://creativecommons.org/licenses/by-nc/3.0/) which permits unrestricted, non-commercial use, distribution and reproduction in any medium, provided the work is properly cited. 